\title{
How to Kill A Virus: Strengthening the Immune System, Reducing Inflammation, Relieving Oxidative Stress, Early Detection in the Prevention and Treatment of SARS- CoV-2 (COVID-19)
}

\author{
Jerry T Thornthwaite*, Daniel Strasser and Larry Loomis \\ Department of Microbiology, Cancer Research Institute of West Tennessee, USA \\ *Corresponding Author: Jerry T Thornthwaite, Department of Microbiology, \\ Cancer Research Institute of West Tennessee, USA.
}

DOI: 10.31080/ASMI.2020.04.0760
Received: November 04, 2020

Published: January 22, 2021

(C) All rights are reserved by Jerry T

Thornthwaite.

\begin{abstract}
The bioavailability of important antiviral compounds, their synergism, and boosting the Natural Killer Cells (NKC) are critical in preventing and treating viral diseases. Based on significant research and clinical studies, molecules and elements are identified that enhance their immune modulator activity, specificallythe NKC. These components include Curcumin, Artemisinin, bioflavonoids, Vitamin D3, N-Acetylcysteine, zinc, and selenium, as shown in this review. Increasing the NKC activity, especially in the immunecompromised elderly population, is critical in developing the first defense line against SARS-CoV-2 (COVID-19). When a patient tests COVID-19 positive, proactive treatment can be accomplished to kill the virus. Early detection with the initial measurement of IgA in the nasal passage using a saliva test is critical in the first detection process. Using an analogy from cancer research, one wants to cure cancer before it metastasizes. With COVID-19, the infection must be stopped in the nose before it "metastasizes" to the lungs. The encapsulation of the above components in micelles (3-10nm in diameter) called NutraNanoSpheres (NNS) are protective spheres around the captured molecules inside using fatty acids with hydrophilic heads on the outside, making them thoroughly water-soluble. Therefore, the NNS may be taken orally with no breakdown in the stomach, resulting in high bioavailability into the bloodstream. The NNS can be introduced as a nasal spray to prevent or treat viral infections in the Nasopharynx. We have developed a line of NNS that bind to the Spike protein, which may lead to the neutralization of viruses and viral infected cells. The NNS binding to the virus may attract the NKC to increase their killing ability. Finally, as we have seen with our malaria studies, we may be able to develop "in vivo immunization" by the breaking up of viral components for introduction to all phases of the immune system.
\end{abstract}

Keywords: SARS-CoV-2; COVID-19; Malaria; NutraNanoSpheres (NNS)

\section{Introduction}

As of now, there is no known effective pharmaceutical agent that cures the SARS-CoV-2 virus. Several antiviral drugs such as ribavirin, remdesivir, lopinavir/ritonavir, antibiotics such as azithromycin and doxycycline, and anti-parasitic drugs such as ivermectin are recommended for COVID-19 treatment. More recent studies have highlighted the possibility of treating patients by the synergistic action of zinc and Hydroxychloroquine in patients suffering from the coronavirus disease.

As shown in figure 1, Severe Acute Respiratory Syndrome Coronavirus 2 (SARS-CoV-2) is the pathogen that causes coronavirus disease recognized in 2019 (COVID-19). The SARS-CoV-2 isan envel- oped, $\beta$-coronavirus, with alarge, positive-sense, single-strandedRNA genome ranging from 26-32 kilobases. The Spike (S) protein, envelope (E) protein, membrane $(\mathrm{M})$ protein, and nucleocapsid $(\mathrm{N})$ protein are the four structural proteins found in the virus. The $S$ protein interacts with the host cell membrane to enable the virus' entry during infection.

This review will focus on the importance of nutraceuticals in the prevention and treatment of COVID-19. The bioavailability of important antiviral compounds, their synergism with each other, the role Natural Killer Cells (NKC) play, and the importance of early detection with the initial measurement of IgA in the nasal passage using a saliva test will be presented. 


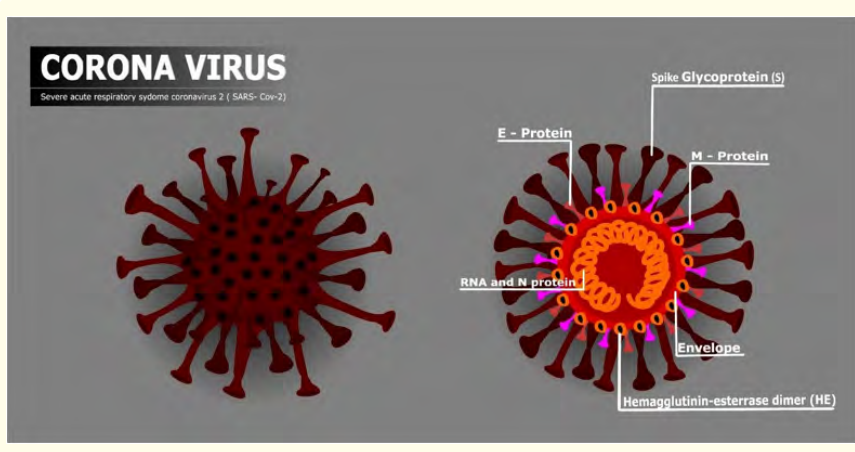

Figure 1: Surface and internal structure of the Corona Virus.

\section{Antiviral components}

The following components are selected based on their antiviral activity, as published in the literature. These nutraceuticals have been micellized in our NutraNanoSpheres ${ }^{\mathrm{TM}}$ (NNS). The NNS are water-soluble and range in diameter between 3-10nm diameter spheres resulting in significantly enhanced bioavailability. The NNS may be prepared in a customized fashion that can be administered easily orally, intravenously, as a mouth wash, and an aerosol spray for the nose. The review of the antiviral components is not exhaustive but, hopefully, thorough enough to show each entity's importance.

Furthermore, the water-soluble nature will make in vitro culture experiments more accessible and more quantitative to perform. The components are chemically defined, reagent grade in the 95-99\% purity range, except for the bioflavonoids, which are about 50\%. These components include the NNS micellized Curcumin, Artemisinin, Vitamin D3, N-Acetylcysteine, Vitamin D3, zinc, selenium, and Bioflavonoids.

\section{Curcumin}

Curcumin targets the Inosine monophosphate dehydrogenase (IMPDH) enzyme involved in the synthesis of guanine nucleotides, thus inhibiting viral replication. Curcumin also shows anti- inflammatory and bronchodilator effects and relieves the respiratory troubles caused by a COVID-19. Curcumin helps to prophylactically stimulate the Natural Killer Cells (NKC), which are the God-given cornerstone in fighting viral diseases. Clinical trials are beginning, which include Curcumin to reduce the risk of the active disease being passed through droplet transmission or direct contact $[1,2]$.

\section{Artemisinin}

Artemisinin is a sesquiterpene lactone with a peroxide moiety that is isolated from the herb Artemisia annua. Artemisinin displays multiple pharmacological actions against inflammation, viral infections, and tumor proliferation. Therefore, Artemisinin is effective against many diseases, while having a relatively safe toxicity profile. Artemisinin is effective against different respiratory disorders, such as lung cancer and inflammatory conditions. In the case of SARS- Cov-2, Artemisinin attenuates inflammation ("cytokine storm") by suppressing the expression of pro-inflammatory cytokines, such as nuclear factor-kappa B (NF-kB), matrix metalloproteinases (MMPs), vascular endothelial growth factor (VEGF), and reactive oxygen species (ROS) production. Patients undergoing respiratory distress have a high concentration of pro- inflammatory cytokines, such as interleukin (IL)-6, which indicates a poor prognosis in COVID- 19.

Furthermore, the infiltration of pro-inflammatory cells, mainly involving macrophages and T- helper cells, has been found in lung tissues of patients with COVID-19. The cytokine storm's clinicalpathological features show that COVID-19 selectively induces a high level of IL- 6 and results in the exhaustion of lymphocytes. The current evidence indicates that Artemisinin is effective against the cytokine storm [3]. Artemisinin targets inflammatory networks, such as NF- $\kappa B$, IRF3, ERK, and ROR $\gamma$ t, which inhibit the differentiation of inflammatory cells and the production of cytokines and chemokines while enhancing IL-10, an anti-inflammatory cytokine $[4,5]$. Interestingly, The World Health Organization (WHO) has noted that the widespread use of Artemisinin Derivative-based therapies may contribute to a lower number of COVID-19 cases than expected [6].

Antimalaria drugs are concentrated 10 to 160 -fold in the lungs of COVID-19 positive patients compared to COVID-19 negative patients [6]. Both Artemisinin and another anti-malaria drug, Hy- 
How to Kill A Virus: Strengthening the Immune System, Reducing Inflammation, Relieving Oxidative Stress, Early Detection in the Prevention and Treatment of SARS- CoV-2 (COVID-19)

droxychloroquine, can act as good inhibitors of the SARS-CoV-2 Spike protein receptor- binding-domain. Using molecular docking studies, these compounds can prevent Lys353 in hACE2 from interacting with the binding hotspot present on the Spike protein $[7,9]$. These anti- malaria drugs are effective antivirals with excellent safety track records in humans and are recommended for treating SARS-CoV-2 patients after successful clinical studies $[9,10]$.

\section{Vitamin $\mathrm{D}_{3}$}

Vitamin D3 reduces the risk of respiratory tract infections through several mechanisms. These include inducing cathelicidins and defensins that can lower viral replication rates and decrease concentrations of pro-inflammatory cytokines that injure the lining of the lungs, leading to pneumonia, as well as increasing concentrations of anti-inflammatory cytokines. Several observational studies and clinical trials have presented evidence of oral dosages of Vitamin D3 in reducing the risk of COVID-19 [11]. One example of the importance of having adequate levels of Vitamin D includes that Corona-type outbreaks peak in winter, a time when the conversion of Vitamin D to Vitamin D3 in the skin cannot occur because of the lack of sunshine. Only $40 \%$ of the US population have adequate Vitamin D3 levels anyway; therefore, vitamin D deficiency contributes to acute respiratory distress syndrome. That case-fatality rates increase with age and with chronic disease comorbidity. To reduce the risk of infection, people at risk of influenza and COVID-19 should consider taking 10,000 IU/d of vitamin D3 for a few weeks, followed by $5000 \mathrm{IU} / \mathrm{d}$. Therefore, Vitamin D treatment is found to decrease viral respiratory tract infection [12-14]. Patients receiving the above high dose of vitamin D supplementation achieved normalization of vitamin D levels and improved clinical recovery evidenced by shorter lengths of stay, lower oxygen requirements, and reduced inflammatory marker status $[15,16]$.

\section{Zinc}

Zinc $(\mathrm{Zn})$ deficiency is a severe public health problem worldwide [17-19]. Several randomized control trials (RCT) have shown that zinc given during an acute episode of diarrhea significantly reduces the duration and risk of persistent disease [20]. The WHO has adopted the addition of $\mathrm{Zn}$ in treating childhood diarrhea. $\mathrm{Zn}$ supplementation reduces the incidence of acute lower respiratory infections in young children [21-23]. In one large RCT from India enrolling young infants with signs of severe bacterial illness, it was investigated whether zinc could reduce the risk of treatment failure [24]. Children taking $\mathrm{Zn}$ had a $40 \%$ reduction in treatment failure from respiratory illness than the placebo group [24]. There have been a significant number of RCT studies on the common cold (a Corona Virus), which showed Zn early supplementation reduced the duration of the disease by 1 to 3 days [25-27].

Sufficient pre-clinical rationale and evidence have been presented to use Chloroquine or Hydroxychloroquine for the treatment of COVID-19. Furthermore, $\mathrm{Zn}$ can enhance innate and adaptive immunity in the course of viral infection [28]. Besides, Zn supplements can favor COVID-19 treatment using those suggested and recommended drugs. Again, the effectiveness of Zn can be enhanced by using Chloroquine or Hydroxychlorquine as an ionophore, while $\mathrm{Zn}$ inside the infected cell can stop SARS-CoV-2 replication $[29,30]$.

Besides the apparent synergism between Zn and Hydroxychloroquine, there are other micronutrient pathways in which $\mathrm{Zn}$ can interfere with viral replication [30]. There is evidence for synergism with zinc, selenium, and Vitamin D3 for resistance to other viral infections, enhancement of immune function, and reduced inflammation. Nutritional intervention, especially for high-risk patients, should be started before administering specific and supportive medical measures [29]. $\mathrm{Zn}$ is an essential cofactor of numerous enzymes $[30,31]$ and can enhance innate and adaptive immunity in the course of viral infection [32-34].

\section{Selenium}

Selenium (Se) is an essential trace element and is bound as selenocysteine in the catalytical centers of many selenoproteins [35-37]. The protective effect of selenium is explained by its role as an essential cofactor in a group of enzymes that, with Vitamin $\mathrm{E}$, reduces the formation of reactive oxygen species (ROS). Besides its importance in the immune system, nutritional deficiencies of selenium are essential in the pathogenicity of viruses [38-40]. The crucial roles that selenoproteins play in regulating reactive oxygen species (ROS) and redox status in nearly all tissues strongly influences inflammation and immune responses. In this way, Se can boost the immune system in aging immunity and protect against viruses and other pathogens [41]. Se deficiency significantly increases the susceptibility to influenza-induced lung pathology associated with pro-inflammatory cytokines [42]. 
How to Kill A Virus: Strengthening the Immune System, Reducing Inflammation, Relieving Oxidative Stress, Early Detection in the Prevention and Treatment of SARS- CoV-2 (COVID-19)

In the case of COVID-19, the main protease responsible for the viral replication interacts with the essential seleno-enzyme glutathione peroxidase1 (GPX1), which is strongly dependent on an adequate selenium supply [40,43]. Furthermore, GPX mimic (a synthetic selenium compound) is a potent inhibitor of the SARS-CoV-2 main protease [44]. Genetic amplification screening of the SARSCoV-2 gene markers provides further proof of protein interactions and antisense transcript mRNA-mRNA interactions occurring at selenocysteine-related insertions in RNA viruses [45]. Therefore, Se supplementation can play a substantial role in inhibiting the replication of the SARS-CoV-2 [46].

Oxidative changes are triggered both in invading microorganisms and in the cells of the host [47]. In the elderly, Se treatment modulates the response to the influenza vaccination, being accompanied by increased IFN- $\gamma$ levels after immunization [48]. Therefore, selenium supplementation in immune-compromised individuals has been considered a safe and effective adjuvant therapy in preventing viral infections [49]. When considering the positive effects of selenium on immune regulation and inflammation in populations low in selenium, it appears justified to use Se as a protective measure against the dramatic, elevated inflammation characterizing corona viral infections. Thus, in subjects with low plasma Se less than $100 \mu \mathrm{g} / \mathrm{L}$, supplementation at a dose of 100-200 $\mu \mathrm{g} \mathrm{Se} /$ day will achieve rapid saturation of vital selenoproteins. Normalization of the selenoproteins should act as an adjuvant approach to prevent aggressive SARS-CoV-2 infection [44-49].

\section{N-Acetyl Cysteine (NAC)}

COVID-19 may cause pneumonia, acute respiratory distress syndrome, cardiovascular alterations, and multiple organ failure, which results in a systemic inflammatory response ("cytokine storm") by an attack from the patient's immune system.

Glutathione is a major endogenous antioxidant that is frequently depleted in COVID-19 patients with chronic obstructive pulmonary disease and acute respiratory distress syndrome. This dramatic deficiency is the result of Glucose-6-phosphate dehydrogenase (G6PD) deficiency in COVID-19 patients resulting in glutathione depletion [50]. $\mathrm{N}$-acetylcysteine (NAC) by oral administration is rapidly converted to cysteine, a precursor to glutathione, leading to significant increases in the patient's glutathione concentrations [51]. Furthermore, NAC is a direct scavenger of ROS, leading to an- tioxidant effects. Administration in vivo leads to anti-inflammatory events (e.g., decreased IL- 6 and TNF alpha concentrations) and antioxidant effects in several pulmonary diseases, including viral pneumonia and acute respiratory distress syndrome [50]. NAC also has activity as an anti-mucolytic due to its ability to disrupt disulfide cross-bounds in the glycoproteins of respiratory mucus.

The systemic inflammation in COVID-19 patients with severe cytokine storms is now making NAC therapy necessary. NAC's oral administration is likely to attenuate the risk of developing COVID-19, as previously demonstrated for influenza and influenza-like illnesses. The thiols from NAC block the angiotensin-converting enzyme 2, thereby hampering penetration of SARS- CoV-2 into cells. COVID-19 patients may have respiratory mucus buildup limiting adequate airflow in the endotracheal tube [50,51]. Systemic or/ and aerosolized NAC may be beneficial in reversing the cytokine storm's effects, causing a reversal of the COVID-19 patient lung complications. G6PD-deficient patient treatment with hydroxychloroquine benefits from intravenous (IV) NAC. NAC blocks hemolysis and elevation of liver enzymes, C-reactive protein (CRP), and ferritin. A full recovery in $90 \%(n=10)$ of G6PD-deficient patients showed a viral infection blockade and prevention of the cytokine storm [52]. Moreover, high-dose intravenous NAC may play an adjuvant role in treating severe COVID-19 cases and in the control of its lethal complications, including pulmonary and cardiovascular adverse events $[53,54]$.

\section{Bioflavonoids}

Safe, efficacious, and inexpensive interventions that have a scientific base should be prioritized for practical use in the current context of the global COVID-19 health pandemic. Bioflavonoids are a group of what are called "polyphenolic" plant-derived compounds. There are between 4,000 and 6,000 different varieties known. Bioflavonoids have been used in nutraceutical medicine to enhance antioxidation, as in the case of Vitamin C. They are also used to support blood circulation, antioxidants and treat allergies, viruses, arthritis, and other inflammatory conditions. The antioxidant power of bioflavonoids is well documented [55-66].

An example of synergism with bioflavonoids is how Vitamin C can interact with bioflavonoids such as Quercetin, a well-known bioflavonoid whose antiviral properties are being investigated for their synergistic antiviral action due to overlapping antiviral and 
How to Kill A Virus: Strengthening the Immune System, Reducing Inflammation, Relieving Oxidative Stress, Early Detection in the Prevention and Treatment of SARS- CoV-2 (COVID-19)

immunomodulatory properties and the capacity of ascorbate to recycle Quercetin, increasing its efficacy. The combination of Vitamin $\mathrm{C}$ and Quercetin are useful for antiviral prevention and treatment as an adjunct to promising pharmacological agents such as Remdesivir or convalescent plasma [55].

Angiotensin-Converting Enzyme receptor (ACE2) importantly is found on the surface of lung cells, which bind effectively to the spike protein, enabling the virus to infect the epithelial cells. Bioflavonoid-based molecules bind with high affinity to the spike protein, helicase, and protease sites on the ACE2 receptor used by the SARS-CoV-2 to infect cells and cause COVID-19 [64]. Recent articles summarize the natural flavonoids with potential efficacy against COVID-19 through ACE2 receptor inhibition [63-66].

Other bioflavonoids that may have prophylactic or therapeutic potential against COVID-19 include Caflanone, Equivir, hesperetin, and myricetin. Caflanone, for example, inhibits virus entry factors, such as ABL-2, cathepsin L, cytokines (IL-1 $\beta$, IL-6, IL-8, Mip-1 $\alpha$, TNF- $\alpha$ ), as well as AXL-2, which facilitates mother-to-fetus transmission of coronavirus [56-60].

Plant-based bioflavonoids increase the beneficial intestinal bacteria that are helpful and play a vital role in enhancing the COVID-19 victim's immunity. Minerals like magnesium and zinc, bioflavonoids, Vitamins C, D and E, and a healthy lifestyle [61], one may prevent various infections, including COVID-19 [55-61].

There were severe panics caused by Severe Acute Respiratory Syndrome (SARS) and Middle- East Respiratory Syndrome-Coronavirus that have laid the groundwork for the bioflavonoid treatment of COVID-19 [61]. The antiviral activity of Herbacetin, Rhoifolin, and Pectolinarin is being found to efficiently block the enzymatic activity of SARS-CoV 3CLpro by inhibiting a 3C- like protease (3CLpro). This flavonoid library to systematically probe inhibitory compounds against SARS-CoV 3Clpro may be useful for treating COVID-19 [62].

The new pharmacology: NutraNanoSpheres ${ }^{\mathrm{TM}}$ (NNS) overview

Currently available antiviral drugs are often associated with limitations such as toxicity and the appearance of drug-resistant strains. Therefore, there is a pressing need to develop safe and more efficient antiviral agents [67]. Furthermore, the ideal antiviral drugs should be safe for prevention and active treatment for various viral infections.

Emerging studies increasingly demonstrate the importance of the nasal passage, throat, and salivary glands as virus replication and transmission sites in early COVID-19 disease. We have found the significant components, Curcumin, Artemisinin, and bioflavonoids or Bilberry, of our TriAntiMal Formulation to be very effective against cancer cells [68,69], malaria [70-72], and, based on the literature and our own experience, possibly viruses.

COVID-19 is highly sensitive to agents that disrupt lipid biomembranes. There has been minimal discussion about the potential role of nasal sprays in preventing and treating viral transmission. We have developed safe and highly purified oral formulations that can boost the Natural Killer Cell (NKC) activity [73] and are crucial for protecting against respiratory illness. Some of these NKC stimulators are significantly antiviral. Therefore, we believe we have the basis of a safe, highly purified antiviral formulation to prevent and treat viral disease, either systemically or as a nasal spray, which can disrupt COVID-19 replication.

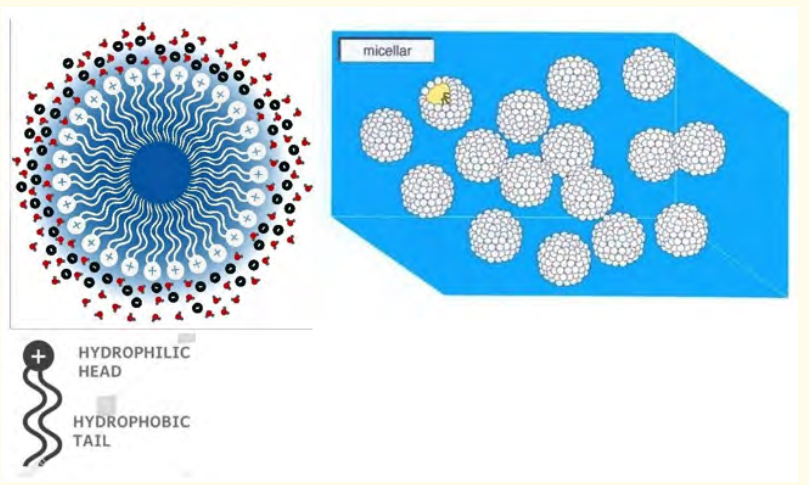

Figure 2: Structure of a Micelle and the resulting sphere products. For example, the micellization of $6 \%$ Curcumin results in bioavailability in the bloodstream many times better than the Curcumin in tablet form. The concentration of the Curcumin NNS is about $8.5 \times 10^{14}$ micelles $/ \mathrm{ml}$. The average diameter is $3-10 \mathrm{~nm}$. 
How to Kill A Virus: Strengthening the Immune System, Reducing Inflammation, Relieving Oxidative Stress, Early Detection in the Prevention and Treatment of SARS- CoV-2 (COVID-19)

To give a size perspective, figure 2 shows the size relation with the NNS compared to other so-called micellized spheres, which can be as large as a thousand nanometers. However, to obtain optimum bioavailability and stability, the NNS must be as small as we can manufacturer them.

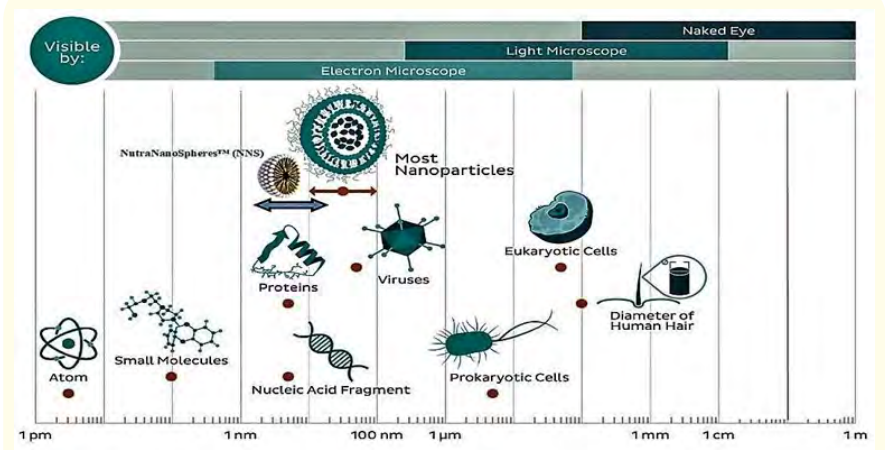

Figure 3: Size perspective of the NutraNanoSpheres as compared to some familiar biological entities. The NNS are 10-100 times smaller than other so-called micellized products. Also, they are 10 to 30 times smaller than COVID-19.

We have a family of nutraceuticals, amino acids, and vitamins already manufactured. Our NNS protect the compounds from breaking down in the stomach acid and allow direct fusion with the intestines for dispersal into the bloodstream. There are no breakdown products that may have potentially dangerous side effects, experienced by a significant number of pharmaceutical drugs.

Since we can protect the compounds from the stomach environment, the oral introduction of peptides, antibodies, and nucleic acid segments are possible with a high bioavailability into the bloodstream. We can produce, for example, oral insulin or B12 without relying on injections.

The NNS are extremely water-soluble and may easily be added to juice or milk using our useful squeeze dropper bottles. We have micellized about 30 nutraceuticals, some of which are combined depending on the health issue. The NNS are heat stable and may be stored at room temperature.
The role of the natural killer cell (NKC)

The Natural Killer Cell (NKC) is the cell-mediated cornerstone of innate immunity (Figure 4). The discovery in 1972, as reviewed (73) of what is now called the Natural Killer Cell (NKC), has helped reveal at least two essential functions of NKCs: Their ability to recognize and kill cancer cells and virally infected cells. The discovery of the NKC was observed by Nomarski optics, scanning (SEM)/ transmission electron microscopy (TEM) with cellular enumeration and enrichment using bovine serum albumin (BSA) continuous gradients. There have been significant research and clinical studies to increase the effectiveness of NKC in the destruction of cancer cells [73].

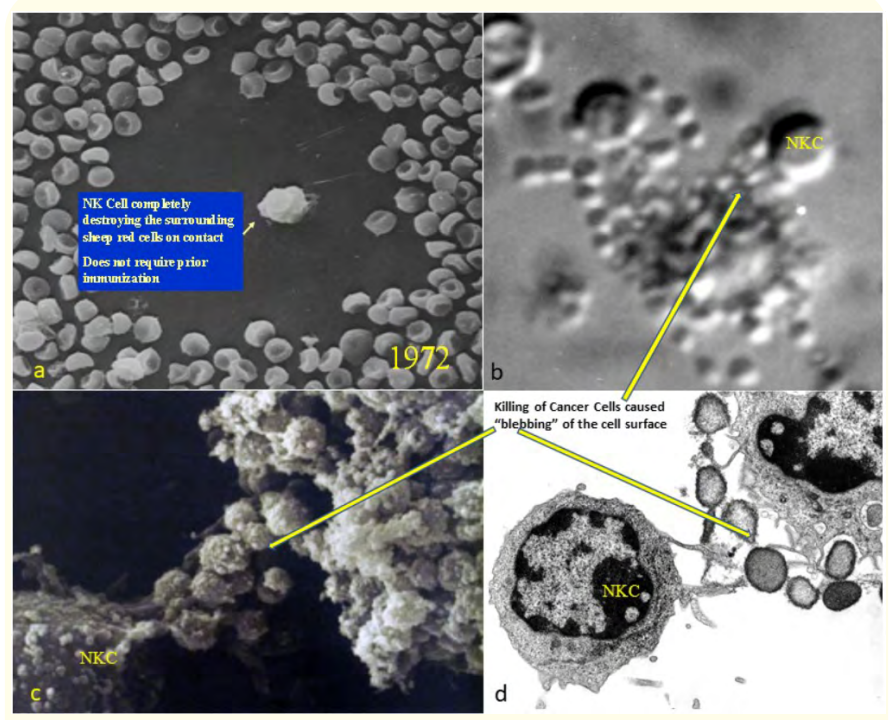

Figure 4: (a) SEM of an NKC destroying foreign sheep red cells; (b) destruction of Cancer Cell by the NKCs as shown by the "Blebbing" of the Cancer cell membranes as shown by Nomarski optics; (c) SEM showing NKC killing a cancer cell; (d) TEM depicting an NKC destroying a cancer cell. The NKC destroys anything that is not itself, such as cancer cells or viral-infected cells, which display their viral antigens on the infected cell's surface.

Based on significant research and clinical studies, at least 16 components have been identified that enhance or may enhance, 
How to Kill A Virus: Strengthening the Immune System, Reducing Inflammation, Relieving Oxidative Stress, Early Detection in the Prevention and Treatment of SARS- CoV-2 (COVID-19)

based on their immune modulator activity, the NKC. These supplements include Alpha Lipoic Acid, Arabinoxylan, Curcumin, Garlic, Genistein, Ginseng, Lentinan, Mistletoe, N-Acetylcysteine, Resveratrol, Selenium, Vitamin B, Vitamin C, Vitamin D3, Vitamin E, and zinc [73]. Increasing the NKC activity in the immune-compromised elderly population is critical in developing the first line of defense against COVID-19.

The role of antimalaria compounds as nutraceuticals for viral disease

The TriAntiMal ${ }^{\mathrm{TM}}$ formulation for curing malaria is ideal for being used as an antiviral as presented in the treatment of using a combination of Curcumin, Artemisinin, and Bilberry to cure malaria and residual viruses. This water-soluble antiviral/malaria version for babies, TriAntiVP ${ }^{\mathrm{TM}}$, describes the three components for malaria being used to treat Viruses (V) and Parasites (P). These nutraceuticals are on the Generally Recognized As Safe (GRAS) list of the FDA and are used to treat malaria in babies as young as two weeks old; therefore, they certainly are safe for the possible prevention and treatment of COVID-19. However, they are NOT FDA approved and are not meant to diagnose, monitor, treat, or cure any viral disease. Since they are on the GRAS list with the FDA, they can go into Phase II trials without the safety trial in Phase I.

The role of antimalaria compounds as nutraceuticals in a nasal spray for treating a viral disease in the nasopharynx

The goal is to use the NutraNanoSphere ${ }^{\mathrm{TM}}$ (NNS) micellized components to develop an easily atomized nasal spray to prevent and treat COVID-19 and any other viruses in the nasal passage. Based on our experience and a significant literature review of enveloped viruses, including coronaviruses, several viral reagents deserve clinical evaluation to reduce transmission of SARS- CoV-2 in the Nasopharynx. Research to test this could include evaluating existing or specifically tailored new formulations in well-designed in vitro viral inactivation assays. Clinical trials on specific population-based interventions with the most at-risk group populations should then be undertaken.

The property of the formulation and the delivery device's design will influence the nasal sprays' dispersion behavior. Determining the droplet size distribution during a spraying treatment is challenging since the actuation is complete in about a tenth of a second. Furthermore, regulatory guidance demands that the particle size of the drug in droplet form must be determined to understand better how a nasal spray product interacts with the body. We have the smallest micellized components in the 3-10 nm diameter range. Compliance with the particle size and bioavailability sets the standard for the nasal spray application and complements these oral administration components. Finally, since our nutraceuticals are on the GRAS list of the FDA, the NNS can be used as a daily preventative and treatment application with minimal concern for toxicity.

The procedures for developing a nasal spray to neutralize the SARS-Co-2 (COVID-19) in the Nasopharynx

- Develop a list of the possible candidates as previously listed.

- $\quad$ Conduct research in well-designed in vitro viral inactivation assays to test each component separately. Since each of the NutraNanoSphere $^{\mathrm{TM}}$ (NNS) components are entirely watersoluble, cell culture testing as a function of the NNS components' concentration can be easily measured.

- The next step would combine NNS components, e.g., of the top three, and test for viral neutralization synergism.

- From the experiment data, the most effective formulation for a nasal spray can be developed.

- Begin clinical studies to determine the safety of administration and follow the virus's nasal passage's clearing.

- Conduct clinical trials in patients using the same combination orally in these patients and evaluate viral clearance in the nasopharynx and the saliva.

- $\quad$ Develop protocols for preventing the SARS-Co-2 virus by using a combination of a prophylactic nasal spray and an oral dose of NNS formulations daily in the most at-risk population groups. 
How to Kill A Virus: Strengthening the Immune System, Reducing Inflammation, Relieving Oxidative Stress, Early Detection in the Prevention and Treatment of SARS- CoV-2 (COVID-19)

Development of an early diagnostic test to measure IgA in saliva

Furthermore, a diagnostic test to measure IgA in saliva would enable very early detection, an average of 60 days before the IgG antibodies are detected. Early detection and treatment of the COVID-19 in the nasopharynx will enable the anti-Covid-19 nasal spray to be used to kill the virus before it infects the lungs. Figure 5 shows the importance of early detection salivary IgA in IgG positive COVID-19 patients. B-cells produce IgA in mucous membranes, the largest antibody class with dimer and secretory forms [74]. The IgG test currently used for detecting COVID-19 is also 91\% positive for IgA in a study of 53 patients [75]. The IgA COVID-19 antibodies have over a 9-fold range of concentration in a study of 83 patients [76]. The average lapse time between salivary IgA positive patient, and the later PCR positive test is 61 days ( $\mathrm{n}=10$; range 32-109 days) [77]. There appears to be a correlation between disease severity and IgA levels $[75,76]$. The COVID-19 IgA appears earliest before IgM about two days after viral exposure $[77,78]$. The secretory IgA may be protective by reducing the possibility of lung involvement [75]. Importantly, a diagnostic test to measure IgA in saliva would enable very early detection, an average of 60 days before the IgG antibodies are detected [77].

Development of a vaccine against COVID-19

The ideal vaccine would include both mucosal IgA and systemic IgG neutralizing antibody production.

Figure 6 shows our approach to a possible immunogen. The NutraNanoSpheres ${ }^{\mathrm{TM}}$ (NNS) may be loaded with spike protein and used as an oral or injectable vaccine. Furthermore, the micelles may be loaded with dinucleotide $\mathrm{CpG}$ or an entity that would activate NKCs to produce cell- mediated immunity against the COVID-19.

There are several ways to facilitate vaccine development. First, the micelles are 10-30 times smaller than the $100 \mathrm{~nm}$ SARS-Co-2 virus (Figure 3). The surface of the NNS may be modified to bind specifically to the spike proteins on the COVID-19. Besides preventing host cell invasion, the cross linking of the NNS with viruses would facilitate an adjuvant effect helping boost the immunization process. Second, the binding of the modified NNS to the spike proteins expressed on the surface of viral infected cells could activate the NKC to destroy the viral infected cells. Third, the NNS could carry CpG to help activate the NKCs even more. Finally, there may be a way to use a family of micellized antivirals to break up the virus and introduce it as an immunogen and have the patient produce their immunity against the virus. We see this with malaria in the form of an "in vivo immunization." The parasite presents its fragments as antigens to the patient's immune system during a daily 16-day treatment, resulting in the patient being immune for years against the Plasmodium falciparum despite repeated assaults by malaria ladened mosquitoes [70-72]. Using the antiviral-NNS taken orally there may be the possiblity that the SARS-Co- 2 could be broken down to present antigens to develop a long-term immunity to the virus.

\section{Conclusions}

Figure 7 is a summary of the ways based on this review of how a virus may be destroyed, leading to long-term immunity.

- NKC activity is increased to destroy viruses and virally infected cells using nutraceuticals.

- NutraNanoSpheres ${ }^{\mathrm{TM}}$ act as carriers of antivirals or therapeutic antigens (e.g., spike proteins, Nano-binding lectins, and Mannose-binding lectins) to facilitate destroying and developing immunity against the COVID-19.

- Use RNA/metabolic Inhibitors against the virus

- Production of therapeutic antibodies which can be delivered by the NNS

- Therapeutic NNS, which have surface markers to attach directly to the spike glycoproteins, may be utilized to prevent their invasion.

The bioavailability of important antiviral compounds, their synergism with each other, boosting the Natural Killer Cells (NKC), the importance of early detection with the initial measurement of IgA in the nasal passage using a saliva test, and the development of "in vivo immunization", as we experienced with malaria, are ways to 
effectively kill viruses "once and for all”. Using an analogy from our cancer research, we try to cure cancer before it metastasizes. With COVID-19, we want to stop it in the nose before it "metastasizes" to the lungs.



Figure 5: Importance of Salivary IgA Immunity in IgG Positive COVID-19 Patients.

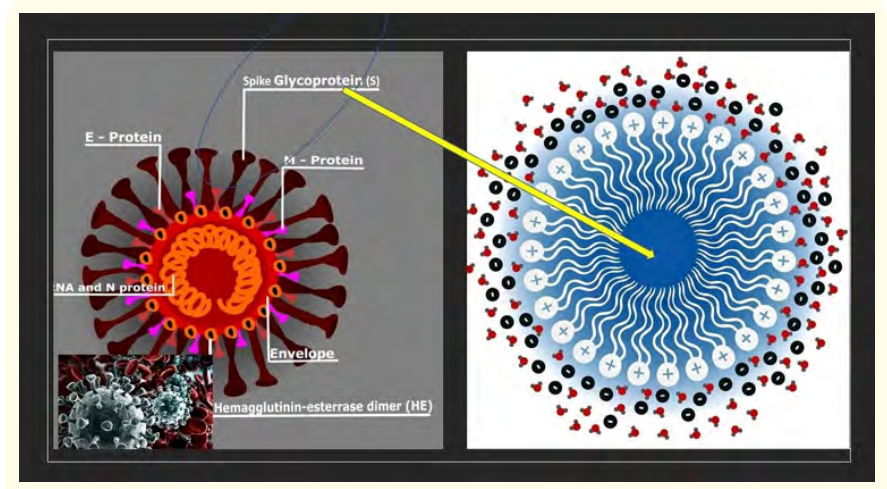

Figure 6: The NutraNanoSpheres ${ }^{\mathrm{TM}}$ may be loaded with spike protein and used as an oral or injectable vaccine. Furthermore, the micelles may be loaded with $\mathrm{CpG}$ dinucleotide or an entity that would activate NKCs to produce cell-mediated immunity against the COVID-19.

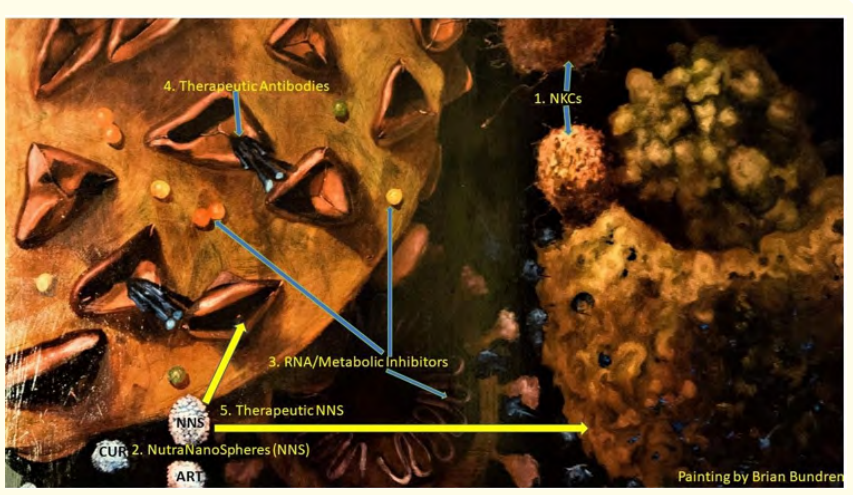

Figure 7: Pathways to the killing of a virus.

\section{Epilogue}

There will be a day soon when the pharmacy will be nearly devoid of bottles with pills. A customer will have their prescription read by a bar code, and a program will dictate the dispensing that will occur. There will be two sections of the pharmacy, each with its dispensing machine. There will be a new pharmacy that will allow an individual to dispense the customized health and wellness products in a droplet, water-soluble form called NutraNanoSpheres $^{\mathrm{TM}}$ (NNS). These NNS are comprised of naturally produced micelles with specific nutraceuticals residing in each nanosphere. of course, there will be premade NNS formulations in squeeze dropper bottles for various health and wellness applications. The other pharmacy section will house the MedNanoSphere ${ }^{\mathrm{TM}}$ formulations, which are custom made by the pharmacist. Depending on the proper dosing formula based on the sex, age, national origin, medical history, allergies, etc., the pharmacist would determine the drop mixtures and duration of the treatment on a customized basis. The side effects that affect the patient and place the pharmaceutical companies into a sea of lawsuits will be greatly minimized. Finally, the nanospheres' incredibly small sizes will allow the passage of much-needed biologics through the skin into the bloodstream while also surviving the stomach if oral dosages are utilized. The stability of the nanosphere products will allow for long shelf lives without the need for refrigeration. Yes, that day is evolving to cure the world's most deadly diseases, including parasitic, bacterial, and viral infections. 
How to Kill A Virus: Strengthening the Immune System, Reducing Inflammation, Relieving Oxidative Stress, Early Detection in the Prevention and Treatment of SARS- CoV-2 (COVID-19)

\section{Acknowledgments}

I thank Dr. Akanni and our Malaria work together; Ken Shumard, Gary Chamblee, Mike Winegrat, Martin Knooce for our Malaria studies in Haiti, Nigeria and Rwanda; Dr. Tony Kirk and Bonita Thornthwaite and Patty O'Neal for their expert writing assistance and laboratory work. I thank Kyle Thornthwaite for his animations and Brian Bundren for the painting in this text. We thank The Shumard Foundation, the Carter Foundation, and Henry Respess for the funding in part of our research. I thank Nathan Clark for serving on our board and providing legal work, Harvey Birnholtz for his accounting assistance, IT guidance by Daniel Simons for the Institute, and my patent attorney Todd Adler. Finally, I am thankful for all of the encouragement from victims and their families who have been affected by the COVID-19.

\section{Bibliography}

1. Burton MJ., et al. "Antimicrobial mouthwashes (gargling) and nasal sprays were administered to patients with suspected or confirmed COVID-19 infection to improve patient outcomes and protect healthcare workers from treating them". Cochrane Database System Reviews 9 (2020): CD013627.

2. Sumathi CS., et al. "The Biological Potentials of Indian Traditional Medicine, Curcumin for Treating Human Diseases". Cardiovascular and Hematological Agents in Medicinal Chemistry (2017).

3. Tang Y., et al. "Cytokine Storm in COVID-19: The Current Evidence and Treatment Strategies". Frontiers in Immunology 11 (2020): 1708.

4. Cheong DHJ., et al. "Anti-malarial drug, Artemisinin and its derivatives for the treatment of respiratory diseases". Pharmacology Research 158 (2020): 104901.

5. Gendrot M., et al. "Antimalarial artemisinin-based combination therapies (ACT) and COVID-19 in Africa: In vitro inhibition of SARS-CoV-2 replication by mefloquine-artesunate". International Journal of Infectious Diseases 99 (2020): 437-440.
6. Sehailia M and Chemat S. "Antimalarial-agent artemisinin and derivatives portray more potent binding to Lys353 and Lys31-binding hotspots of SARS-CoV-2 spike protein than Hydroxychloroquine: potential repurposing of artenimol for COVID-19". Journal of Biomolecular Structure and Dynamics 22 (2020): 1-11.

7. Cao R., et al. "Anti-SARS-CoV-2 Potential of Artemisinins In Vitro". ACS Infection Disease 6.9 (2020): 2524-2531.

8. Uzun T and Toptas O. "Artesunate: could be an alternative drug to chloroquine in COVID-19 treatment? Chinese Medicine 15 (2020): 54 .

9. Devaux CA., et al. "New insights on the antiviral efects of chloroquine against coronavirus: what to expect for COVID19?" International Journal of Antimicrobial Agents (2020).

10. Aldieri E., et al. "Artemisinin inhibits inducible nitric oxide synthase and nuclear factor NF-kB activation". FEBS Letter 552 (2003): 141-144.

11. Meltzer DO., et al. "Association of Vitamin D Deficiency and Treatment with COVID-19 Incidence". medRxiv 13 (2020): 2020.05.08.20095893.

12. Baktash V., et al. "Vitamin D status and outcomes for hospitalised older patients with COVID-19". Postgraduate Medical Journal (2020): postgradmedj-2020-138712.

13. Goddek S. "Vitamin D3 and $\mathrm{K} 2$ and their potential contribution to reducing the COVID-19 mortality rate". International Journal of Infectious Diseases 99 (2020): 286-290.

14. Ilie PC., et al. "The role of vitamin D in the prevention of coronavirus disease 2019 infection and mortality". Aging Clinical and Experimental Research 32.7 (2020): 1195-1198.

15. Ohaegbulam KC., et al. "Vitamin D Supplementation in COVID-19 Patients: A Clinical Case Series". American Journal of Therapy 27.5 (2020): e485-e490.

16. Quesada-Gomez JM., et al. "Vitamin D receptor stimulation to reduce acute respiratory distress syndrome (ARDS) in patients with coronavirus SARS-CoV-2 infections: Revised Ms SBMB 2020_166". The Journal of Steroid Biochemistry and Molecular Biology 202 (2020): 105719. 
17. Wessells KR and Brown KH. "Estimating the global prevalence of zinc deficiency: Results based on zinc availability in national food supplies and the prevalence of stunting". PLOS ONE 7 (2012): e50568.

18. Black RE. Zinc deficiency, infectious disease and mortality in the developing world". Journal of Nutrition 133 (2003): 1485s-1489s.

19. Bhutta ZA. "Iron and zinc deficiency in children in developing countries”. BMJ (Clin. Res. ed.) 334 (2007): 104-105.

20. Lazzerini M and Wanzira H. "Oral zinc for treating diarrhoea in children". Cochrane Database System Reviews 12 (2016): CD005436.

21. Skalny AV., et al. "Zinc and respiratory tract infections: Perspectives for COVID19 (Review)". International Journal of Molecular Medicine 46 (2020): 17-26.

22. Lassi ZS., et al. "Zinc supplementation for the prevention of pneumonia in children aged 2 months to 59 months". Cochrane Database System Reviews 12 (2016): CD005978.

23. Haider BA., et al. "Zinc supplementation as an adjunct to antibiotics in the treatment of pneumonia in children 2 to 59 months of age". Cochrane Database System Reviews (2011).

24. Bhatnagar S., et al. "Zinc as adjunct treatment in infants aged between 7 and 120 days with probable serious bacterial infection: A randomised, double-blind, placebo-controlled trial". Lancet 379 (2012): 2072-2078.

25. Science M., et al. "Zinc for the treatment of the common cold: A systematic review and meta-analysis of randomized controlled trials". CMAJ 184 (2012): E551-E561.

26. Hemila H. "Zinc lozenges may shorten the duration of colds: A systematic review". Open Respiratory Medicine Journal 5 (2011): 51-58.

27. Basnet S., et al. "Oral zinc and common childhood infectionsAn update". Journal of Trace Elements in Medicine and Biology 31 (2015): 163-166.
28. Shittu MO and Afolami OI. "Improving the efficacy of Chloroquine and Hydroxychloroquine against SARS-CoV-2 may require Zinc additives - A better synergy for future COVID-19 clinical trials". Infez Med 28.2 (2020): 192-197.

29. Alexander J., et al. "Early Nutritional Interventions with Zinc, Selenium and Vitamin D for Raising Anti-Viral Resistance Against Progressive COVID-19”. Nutrients 12.8 (2020): 2358.

30. Rahman MT and Idid SZ. "Can Zn Be a Critical Element in COVID-19 Treatment?" Biological Trace Element Research 26 (2020): 1-9.

31. Tainer JA., et al. "Structure and mechanism of copper, zinc superoxide dismutase". Nature 306 (1983): 284-287.

32. Maares M and Haase H. "Zinc and immunity: An essential interrelation". Archives of Biochemistry and Biophysics 611 (2016): 58-65.

33. Tuerk MJ and Fazel N. "Zinc deficiency". Current Opinion in Gastroenterology 25 (2009): 136-143.

34. Kulik L., et al. "Zinc deficiency drives Th17 polarization and promotes loss of Treg cell function". Journal of Nutritional Biochemistry 63 (2019): 11-18.

35. Fairweather-Tait SJ., et al. "Selenium in human health and disease". Antioxidants and Redox Signaling 14 (2011): 1337-1383.

36. Beck MA and Matthews CC. "Micronutrients and host resistance to viral infection". Proceedings of the Nutrition Society 59 (2000): 581-585.

37. Long J., et al. "Dietary Serine Supplementation Regulates Selenoprotein Transcription and Selenoenzyme Activity in Pigs". Biological Trace Element Research (2020).

38. Zhang J., et al. "Association between regional selenium status and reported outcome of COVID-19 cases in China". The American Journal of Clinical Nutrition 111 (2020): 1297-1299.

39. Moghaddam A., et al. "Selenium Deficiency Is Associated with Mortality Risk from COVID-19”. Nutrients 12 (2020): 2098. 
How to Kill A Virus: Strengthening the Immune System, Reducing Inflammation, Relieving Oxidative Stress, Early Detection in the Prevention and Treatment of SARS- CoV-2 (COVID-19)

40. Gordon DE., et al. "A SARS-CoV-2 protein interaction map reveals targets for drug repurposing". Nature 583 (2020): 459-468.

41. Avery JC., et al. "Selenium, Selenoproteins, and Immunity". Nutrients 10 (2018): 1203.

42. Beck MA., et al. "Selenium deficiency increases the pathology of an influenza virus infection". FASEB Journal 15 (2001): 1481-1483.

43. Seale LA., et al. "A role for selenium-dependent GPX1 in SARSCoV-2 virulence". The American Journal of Clinical Nutrition (2020).

44. Sies $\mathrm{H}$ and Parnham MJ. "Potential therapeutic use of ebselen for COVID-19 and other respiratory viral infections". Free Radical Biology and Medicine 156 (2020): 107-112.

45. Vavougios GD. "Selenium-Associated gene signatures within the SARS-CoV-2-Host genomic interaction interface". Free Radical Biology and Medicine (2020).

46. Pearson H., et al. "SARS: What have we learned?" Nature 424 (2003): 121-126.

47. Harthill M. "Review: Micronutrient selenium deficiency influences evolution of some viral infectious diseases". Biological Trace Element Research 143 (2011): 1325-1336.

48. Ivory K., et al. "Selenium supplementation has beneficial and detrimental effects on immunity to influenza vaccine in older adults". Clinical Nutrition 36 (2017): 407-415.

49. Steinbrenner H., et al. "Dietary selenium in adjuvant therapy of viral and bacterial infections". Advances in Nutrition 6 (2015): 73-82.

50. Sadowska AM., et al. "Antioxidant and anti-inflammatory efficacy of NAC in the treatment of COPD: discordant in vitro and in vivo dose-effects: a review". Pulmonary Pharmacology and Therapeutics 20.1 (2007): 9-22.

51. Szakmany T., et al. "N-acetylcycteine for sepsis and systemic inflamatory response in adults". Cochrane Database of Systemic Reviews 9 (2012): CD006616.
52. Ibrahim H., et al. "Therapeutic blockade of inflammation in severe COVID-19 infection with intravenous N- acetylcysteine". Clinical Immunology 219 (2020): 108544.

53. De Flora S., et al. "Rationale for the use of $\mathrm{N}$-acetylcysteine in both prevention and adjuvant therapy of COVID-19". FASEB Journal 11 (2020): 10.1096/fj.202001807.

54. Horowitz RI and Freeman PR. "Three novel prevention, diagnostic, and treatment options for COVID- 19 urgently necessitating controlled randomized trials". Medical Hypotheses 143 (2020): 109851.

55. Colunga Biancatelli RML., et al. "Quercetin and Vitamin C: An Experimental, Synergistic Therapy for the Prevention and Treatment of SARS-CoV-2 Related Disease (COVID-19)". Frontiers in Immunology 11 (2020): 1451.

56. Ngwa W., et al. "Potential of Flavonoid-Inspired Phytomedicines against COVID-19". Molecules 25.11 (2020): 2707.

57. Russo M., et al. "Roles of flavonoids against coronavirus infection". Chemico-Biological Interactions 328 (2020): 109211.

58. Arshad MS., et al. "Coronavirus Disease (COVID-19) and Immunity Booster Green Foods: A Mini Review". Food Science and Nutrition 8.8 (2020): 3971-3976.

59. Arshad MS., et al. "Coronavirus Disease (COVID-19) and Immunity Booster Green Foods: A Mini Review". Food Science and Nutrition 8.8 (2020): 3971-3976.

60. Tutunchi H., et al. "Naringenin, a flavanone with antiviral and anti-inflammatory effects: A promising treatment strategy against COVID-19". Phytotherapy Research (2020).

61. Alkhatib A. "Antiviral Functional Foods and Exercise Lifestyle Prevention of Coronavirus". Nutrients 12.9 (2020): E2633.

62. Jo S., et al. "Inhibition of SARS-CoV 3CL protease by flavonoids". Journal of Enzyme Inhibition and Medicinal Chemistry 35.1 (2020): 145-151.

63. Muchtaridi M., et al. "Natural Flavonoids as Potential Angiotensin-Converting Enzyme 2 Inhibitors for Anti-SARS-CoV-2". Molecules 25.17 (2020): 3980. 
64. Williamson G and Kerimi A. "Testing of natural products in clinical trials targeting the SARS-CoV-2 (Covid-19) viral spike protein-angiotensin converting enzyme-2 (ACE2) interaction". Biochemical Pharmacology 178 (2020): 114123.

65. Pandey P., et al. "Targeting SARS-CoV-2 spike protein of COVID-19 with naturally occurring phytochemicals: an in silico study for drug development". Journal of Biomolecular Structure and Dynamics 22 (2020): 1-11.

66. Solnier J and Fladerer JP. "Flavonoids: A complementary approach to conventional therapy of COVID-19?" Phytochemical Review 18 (2020): 1-23.

67. Ghaffari H., et al. "Inhibition of H1N1 influenza virus infection by zinc oxide nanoparticles: another emerging application of nanomedicine". Journal of Biomedical Science 26.1 (2019): 70.

68. Thibado SP., et al. "Anticancer effects of Bilberry anthocyanins compared with NutraNanoSphere encapsulated Bilberry anthocyanins". Molecular and Clinical Oncology 8.2 (2018): 330335.

69. Thornthwaite JT., et al. "Anticancer Effects of the Curcumin, Artemisinin, Genistein, and Resveratrol, and Vitamin C: Free vs. Liposomal Forms". Advances in Biological Chemistry 7 (2017): 27-41.

70. Olufemi EO., et al. "Antimalarial Treatment in South- Western Nigeria". Microbiology and Infectious Disease 3 (2019): 1-7.

71. Thornthwaite JT., et al. "DNA Gene Expression to Study Immunologic Mechanisms for the Long-term Cure of Malaria in Babies and Children in South-Western Nigeria". Advances in Biological Chemistry 9: (2019): 1-20.

72. Thornthwaite JT and Akanni EO. "Treating Malaria "Once and for All”. Journal of Immunology and Immunotherapy 2 (2019): 003.

73. Thornthwaite JT., et al. "The Natural Killer Cell: Historical Perspective and the use of Supplements to enhance NKC Activity". Journal of Immune Based Therapies, Vaccines and Antimicrobials 1 (2012): 21-51.
74. Pilette C., et al. "Lung mucosal immunity: immunoglobulin-A revisited”. European Respiratory Journal 18 (2001): 571-580.

75. Varadhachary A., et al. "Salivary anti-SARS-CoV-2 IgA as an accessible biomarker of mucosal immunity against COVID-19". medRxiv (2020).

76. Hansen CB., et al. "SARS-CoV-2 antibody responses determine disease severity in COVID-19 infected individuals". BioRxiv (2020).

77. Ma H., et al. "Serum IgA, IgM, and IgG responses in COVID-19". Cellular and Molecular Immunology 17 (2020): 773-775.

78. Yu H., et al. "Distinct features of SARS-CoV-2-specific IgA response in COVID-19 patients". European Respiratory Journal (2020): 2001526.

\section{Assets from publication with us}

- Prompt Acknowledgement after receiving the article

- Thorough Double blinded peer review

- Rapid Publication

- Issue of Publication Certificate

- High visibility of your Published work

Website: www.actascientific.com/

Submit Article: www.actascientific.com/submission.php

Email us: editor@actascientific.com

Contact us: +919182824667 